\title{
Efectividad de enmiendas sobre el crecimiento y rendimiento de fréjol común en suelo andisol ácido
}

\section{Efectivity of amendments on growth and yield of common beans in an acidic andisol soil}

\author{
Anabel del Carmen Cuenca-Tinoco ${ }^{1 *} \oplus$; Noemi Isabel Castro-Delgado ${ }^{\circledR}$; Jessica Elizabeth Cargua-Chávez ${ }^{1 \oplus}$ \\ Galo Alexander Cedeño-García ${ }^{2}$; José Luis Cedeño-Zambrano ${ }^{3 \odot}$
}

Recibido para publicación: 30 de marzo de 2020 - Aceptado para publicación: 27 de mayo de 2020

\section{RESUMEN}

El objetivo de la investigación fue evaluar el efecto de varias enmiendas sobre el crecimiento $y$ rendimiento del fréjol común en un suelo andisol ácido de la zona de Santo Domingo de los Tsáchilas, Ecuador. Los tratamientos evaluados fueron $\mathrm{T}_{1}$ (Humus + harina de rocas), $\mathrm{T}_{2}$ (Humus + dolomita), $\mathrm{T}_{3}$ (Compost + harina de rocas), $\mathrm{T}_{4}$ (Compost + dolomita), $\mathrm{T}_{5}$ (Gallinaza + harina de rocas), $\mathrm{T}_{6}$ (Gallinaza + dolomita), $\mathrm{T}_{7}$ (Fertilización convencional) y $\mathrm{T}_{8}$ (Control). El diseño utilizado fue de bloques completos al azar con tres repeticiones. Las principales variables registradas fueron peso seco de planta (g), rendimiento de grano $\left(\mathrm{kg} \mathrm{ha}^{-1}\right)$ y $\mathrm{pH}$ del suelo. Los tratamientos de enmiendas influenciaron significativamente $(p<0,05)$ el peso seco de planta, el rendimiento de grano y el $\mathrm{pH}$ del suelo. Los tratamientos más efectivos fueron las combinaciones de dolomita + gallinaza, compost $y$ humus, que incrementaron el crecimiento en términos de peso seco en 22, 27 y 31\%, respectivamente, en relación al tratamiento control. El mayor incremento en rendimiento de granos también fue alcanzado con dolomita + gallinaza, compost y humus, con 21, 28 y $30 \%$, respectivamente, en relación al tratamiento testigo. El pH del suelo fue elevado hasta 5,74; 5,76 y 5,85 con dolomita + gallinaza, compost y humus, respectivamente. La aplicación de enmiendas alcalinizantes debe ser una práctica común para potenciar crecimiento, rendimiento y respuesta a la fertilización del frejol común en suelos andisoles ácidos.

Palabras clave: Desarrollo; Encalado; Producción; Phaseolus vulgaris.

\footnotetext{
${ }^{1}$ Instituto Superior Tecnológico Calazacón, Santo Domingo de los Tsáchilas, Ecuador.

2 Escuela Superior Politécnica Agropecuaria de Manabí Manuel Félix López, Manabí, Ecuador.

${ }^{3}$ Universidad UTE, Santo Domingo de los Tsáchilas, Ecuador.

* Autor para correspondencia: M.Sc. Anabel del Carmen Cuenca-Tinoco

Email: a.cuencaistc@gmail.com
}

\begin{abstract}
The main objective of the research was to evaluate the effect of various amendments on the growth and yield of common beans in an acidic andisol soil in the Santo Domingo de los Tsáchilas area, Ecuador. The treatments evaluated were $\mathrm{T}_{1}$ (Humus + rock flour), $\mathrm{T}_{2}$ (Humus + dolomite), $\mathrm{T}_{3}$ (Compost + rock flour), $\mathrm{T}_{4}$ (Compost + dolomite), $\mathrm{T}_{5}$ (Gallinaza + rock flour), $\mathrm{T}_{6}$ (Gallinaza + dolomite), T7 (Conventional Fertilization) and T8 (Control). The design used was randomized complete blocks with three repetitions. The main variables recorded were plant dry weight $(\mathrm{g})$, grain yield $\left(\mathrm{kg} \mathrm{ha}^{-1}\right)$ and soil $\mathrm{pH}$. The amendment treatments significantly influenced $(p<0,05)$ the plant dry weight, grain yield and soil $\mathrm{pH}$. The most effective treatments were the combinations of dolomite + chicken manure, compost and humus, which increased growth in terms of dry weight by 22, 27 and $31 \%$, respectively, in relation to the control treatment. The greatest increase in grain yield was also achieved with dolomite + chicken manure, compost and humus, with 21,28 and $30 \%$, respectively, in relation to the control treatment. The soil $\mathrm{pH}$ was raised to 5,74; 5,76 and 5,85 with dolomite + chicken manure, compost and humus, respectively. The application of alkalizing amendments should be a common practice to enhance growth, yield and response to fertilization of common beans in acidic andisol soils.
\end{abstract}

Keywords: Development; Production; Liming; Phaseolus vulgaris.

\section{Cómo citar}

Cuenca-Tinoco, A. d. C., Castro-Delgado, N.I., Cargua-Chávez, J.E., Cedeño-García, G.A. y Cedeño-Zambrano, J.L. 2020. Efectividad de enmiendas sobre el crecimiento y rendimiento de fréjol común en suelo andisol ácido. Temas Agrarios 25(1): 54-65 https://doi.org/10.21897/rta.v25i1.2236

Temas Agrarios 2020. Este artículo se distribuye bajo los términos de la Licencia Creative Commons Attrubution 4.0 (https://creativecommons.org/licenses/by-nc/4.0/deed.es), que permite copiar, redistribuir, remezclar, transformar y crear a partir del' material, de forma no
comercial, dando crédito y licencia de forma adecuada a los autores de la obra.

Temas Agrarios. Enero - Junio 2020; Vol. 25. No. 1, pp. 54-65| ISSNe 2389-9182 | https://doi.org/10.21897/rta.v25i1.2236 


\section{INTRODUCCIÓN}

El fréjol común (Phaseolus vulgaris L.) es la leguminosa más importante para la alimentación de más de 300 millones de personas, la mayoría de ellas en países en vía de desarrollo (Alemu, 2017). A nivel mundial se está valorando cada vez con mayor importancia a esta leguminosa, dado el alto contenido en hierro y polifenoles con capacidad antioxidante considerados de gran importancia en la salud humana (Petry et al., 2015; Yang et al., 2018). En Ecuador es una de las leguminosas más cultivadas bajo sistemas de producción de monocultivo y asociada, y es consumido tanto como vainas inmaduras y grano seco, debido a su alto contenido proteínico se considera al frejol como un rubro básico de la canasta familiar que contribuye a la seguridad alimentaria del país (Peralta et al., 2013; MAG, 2017).

Si bien el cultivo representa un alto valor alimentario en la dieta diaria de la sociedad, su producción se ve afectada por algunos factores bióticos y abióticos. Entre los factores abióticos, la acidez del suelo afecta negativamente la producción de frejol a través de diferentes mecanismos, incluida la toxicidad del aluminio y la inhibición de la disponibilidad de nutrientes esenciales (N, P, K) para el cultivo (Alemu, 2017). El bajo pH y la toxicidad de aluminio restringe el crecimiento y el alargamiento de las raíces, además de reducir significativamente el rendimiento del cultivo (Legesse et al., 2013; Lal, 2016).

De acuerdo con Mite et al. (2009) en el litoral ecuatoriano se ubican zonas productoras con suelos volcánicos ácidos, donde las elevadas precipitaciones (> $3000 \mathrm{~mm}$ anuales) contribuyen con altos niveles de lavado de bases, disminución de la $\mathrm{CIC}$, pH y alta concentración de $\mathrm{Al}+3$ en la solución del suelo, lo cual disminuye el potencial de rendimiento de los cultivos sensibles. En frejol se ha considerado que el $\mathrm{pH}$ ideal se sitúa entre 6 a 7,5 y que valores menores de 5,0 es crítico para el cultivo debido a que se disminuye la nodulación y producción (Lunze et al., 2007; Shanka et al., 2018). En este sentido, el frejol común es relativamente más sensible a la toxicidad de $\mathrm{Al}+3$ en relación a otras especies, dado que se ha documentado reducciones de rendimiento de entre $30-60 \%$ a causa de este factor edáfico (Rangel et al., 2007; Habtamu, 2017).

Resultados de varias investigaciones demostraron que bajo condiciones de suelos ácidos, la aplicación de enmiendas orgánicasminerales disminuyó la acidez del suelo, mejoró el crecimiento y producción del frejol (Dida y Etisa, 2019). En este sentido, Shanka et al. (2018) reportaron que la aplicación conjunta de cal, compost y fosforo mejoró la nodulación, crecimiento y producción del frejol común en un suelo ácido del sur de Etiopía. Resultados similares fueron reportados por Fekadu et al. (2018) en suelos ácidos de las tierras altas de Etiopía, donde la aplicación combinada de fósforo, estiércol de corral y cal mejoro la producción del haba. En otro estudio similar desarrollado en Nigeria, se reportó que la aplicación combinada de cal y estiércol de corral mejoró notablemente el crecimiento y los componentes de rendimiento del frejol común establecido en suelos ácidos con pH 3.0 (Effa et al., 2019).

Es preciso indicar que no existen estudios similares realizados en el cultivo de frejol común en este tipo de suelo, razón por la cual el objetivo de la investigación fue evaluar la efectividad de enmiendas sobre el crecimiento y rendimiento de fréjol común en un suelo andisol ácido de Ecuador.

\section{MATERIALES Y MÉTODOS}

\section{Localización}

El experimento se desarrolló durante la temporada lluviosa del 2019, en la granja 
experimental Mishili del Instituto Superior Tecnológico Calazacón, localizada en el km 61/2 de la vía Santo Domingo - Quevedo, en la provincia de Santo Domingo de los Tsáchilas, Ecuador. La granja se ubica geográficamente en las coordenadas UTM X: 699427.5 y Y:
9966768.2, a una altitud de 585 msnm, con temperatura promedio anual de $24.9{ }^{\circ} \mathrm{C}$, precipitación promedio de $3052 \mathrm{~mm}$ anuales y heliofanía de 780 horas año-1. En la tabla 1 se resumen las características químicas de suelo del sitio experimental.

Tabla 1. Características químicas del suelo en el sitio experimental. Santo Domingo, Ecuador.

\begin{tabular}{|c|c|c|c|c|c|c|c|c|}
\hline $\mathrm{pH}$ & $\mathrm{MO}$ & $\mathrm{NH} 4$ & $P$ & $S$ & K & $\mathrm{Ca}$ & $M g$ & $\mathrm{~A}+\mathrm{H}$ \\
\hline & $\%$ & \multicolumn{3}{|c|}{----------- $\mathrm{mg} \mathrm{kg}^{-1}$--------- } & \multicolumn{4}{|c|}{ 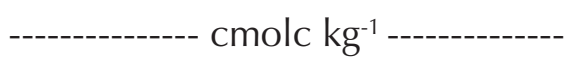 } \\
\hline 4,9 & 4,8 & 27,1 & 7,9 & 3,2 & 0,3 & 4,0 & 0,56 & 1,15 \\
\hline
\end{tabular}

\section{Tratamientos, diseño y unidad experimental}

Como material vegetal se utilizó el cultivar de frejol común arbustivo INIAP 473 - Boliche, el mismo que fue liberado por el Instituto Nacional de Investigaciones Agropecuarias - INIAP para el litoral ecuatoriano. Las principales características de la variedad son grano color rojo, habito de crecimiento determinado, un ciclo de siembra a cosecha de 65 - 73 días, y un potencial de rendimiento de grano de $2224 \mathrm{~kg} \mathrm{ha}^{-1}$. Los tratamientos probados fueron mezclas de enmiendas orgánicas y minerales, las que se describen a continuación y en la tabla 2:

T1: Humus (1,5 $\left.\mathrm{t} \mathrm{ha}^{-1}\right)+$ harina de rocas $\left(1,5\right.$ tha $\left.^{-1}\right)$

T2: Humus (1,5 t ha-1) + dolomita $\left(1,5 \mathrm{t} \mathrm{ha}^{-1}\right)$

T3: Compost $\left(1,5 \mathrm{t} \mathrm{ha}^{-1}\right)+$ harina de rocas $\left(1,5 \mathrm{tha}^{-1}\right)$

T4: Compost (1,5 tha $\left.{ }^{-1}\right)+$ dolomita $\left(1,5 \mathrm{t} \mathrm{ha}^{-1}\right)$

T5: Gallinaza $\left(1,5 \mathrm{t} \mathrm{ha}^{-1}\right)+$ harina de rocas $\left(1,5\right.$ t ha $\left.^{-1}\right)$

T6: Gallinaza $\left(1,5 \mathrm{t} \mathrm{ha}^{-1}\right)+$ dolomita $\left(1,5 \mathrm{t} \mathrm{ha}^{-1}\right)$

T7: Fertilizante compuesto (200 $\left.\mathrm{kg} \mathrm{ha}^{-1}\right)$

T8: Control

Tabla 2. Características químicas de las enmiendas evaluadas en frejol común. Santo Domingo, Ecuador.

\begin{tabular}{|c|c|}
\hline Enmiendas & Características químicas y carga mineral \\
\hline Dolomita & $\mathrm{CaO}(42 \%), \operatorname{MgO}(20 \%)$, Si $(1,1 \%), \mathrm{CIC}\left(5 \mathrm{cmolc}^{-1}\right), \mathrm{pH}(8)$ \\
\hline Harina de Rocas & $\begin{array}{l}\mathrm{N}(0,2 \%), \mathrm{P}_{2} \mathrm{O} 5(3 \%), \mathrm{K} 2 \mathrm{O}(0,3 \%), \mathrm{CaO}(24 \%), \mathrm{MgO}(12 \%), \mathrm{S}(12 \%), \\
\mathrm{Si}(23 \%), \text { Núcleos y micronutrientes }(5 \%), \mathrm{CIC}\left(23 \mathrm{cmolc} \mathrm{kg}^{-1}\right), \mathrm{pH}(7,5)\end{array}$ \\
\hline Gallinaza & $\begin{array}{l}\mathrm{N}(3,3 \%), \mathrm{P}_{2} \mathrm{O} 5(3,1 \%), \mathrm{K} 2 \mathrm{O}(2,1 \%), \mathrm{Ca}(6,1 \%), \mathrm{Mg}(0,8 \%), \text { Micro } \\
\text { elementos y sales }(5,6 \%), \mathrm{MO}(70 \%), \mathrm{pH}(7,2)\end{array}$ \\
\hline Compost & $\begin{array}{l}\mathrm{N}(2 \%), \mathrm{P}_{2} \mathrm{O} 5(2 \%), \mathrm{K} 2 \mathrm{O}(1 \%), \mathrm{CaO}(4 \%), \mathrm{MgO}(1 \%), \mathrm{S}(2 \%), \\
\text { Micronutrientes }(0,08 \%), \mathrm{MO}(35 \%), \mathrm{pH}(8,8)\end{array}$ \\
\hline Humus & $\begin{array}{l}\text { Ácido húmico (59\%), ácido fulvico (42\%), } \mathrm{MO}(33 \%), \mathrm{P}_{2} \mathrm{O} 5(0,1 \%), \mathrm{K} 2 \mathrm{O} \\
(8 \%), \mathrm{CaO}(2 \%), \mathrm{MgO}(0,3 \%), \mathrm{S}(0,3 \%), \text { Micronutrientes }(0,5 \%), \mathrm{pH}(8)\end{array}$ \\
\hline ertilizant & $\mathrm{N}(12 \%), \mathrm{P}_{2} \mathrm{O} 5(11 \%), \mathrm{K} 2 \mathrm{O}(18 \%), \mathrm{MgO}(2,7 \%)$, Micronutrientes $(0,25 \%)$ \\
\hline
\end{tabular}


Se utilizó un diseño de bloques completos al azar con ocho tratamientos, tres repeticiones y 24 unidades experimentales, que se conformaron de parcelas de $25 \mathrm{~m}^{2}$, donde el registro de datos se realizó en las plantas centrales, dejando como efecto borde dos hileras de plantas por cada lado de la parcela experimental. La densidad de siembra utilizada fue 200.000 plantas ha-1, distanciadas a 0,50 m entre hileras y $0,20 \mathrm{~m}$ entre plantas, con dos semillas por sitio de siembra.

Los tratamientos de enmiendas fueron incorporados al suelo 30 días previos a la siembra, excepto el tratamiento con fertilización convencional, donde el fertilizante fue aplicado a la emergencia y 20 días después de la siembra.

\section{Variables respuesta y análisis de datos}

Las variables evaluadas fueron: altura de planta (AP) que se determinó en $\mathrm{cm}$ a los 60 días después la siembra (DDS), para lo cual se registró el dato desde el nivel del suelo hasta la última inserción de trifolios. Longitud radical (LR) que fue determinada en $\mathrm{cm}$ a los 60 DDS, registrando el dato desde el nivel del cuello del tallo hasta el ápice del sistema radical. El peso seco (PS) de planta fue registrado en $g$ a los 60 DDS, para lo cual las plántulas fueron secadas en estufa de aire forzado a $80^{\circ} \mathrm{C}$ hasta alcanzar peso constante, según la metodología descrita por Pérez et al. (2013). El área foliar (AF) fue registrada en $\mathrm{cm}^{2}$ a los 60 DDS mediante el método del sacabocado, que consistió en extraer de las láminas foliares cuadrantes de un área y peso seco conocido, que luego se relacionó con el peso seco de las hojas para obtener el área foliar por planta.

Los componentes de rendimiento como número de vainas planta $^{-1}$, peso de 100 vainas y de 100 granos fueron registrados a la cosecha, para lo cual fueron tomadas al azar 10 plantas del centro de la parcela experimental.
El rendimiento de grano a cosecha $\left(\mathrm{kg} \mathrm{ha}^{-1}\right)$ fue estimado mediante un cuadrante lanzado al azar en el centro de la parcela, donde se registró el peso de granos $\mathrm{m}^{2}$ y con ese dato se cuantifico el rendimiento por hectárea. Además, se cuantificó la variación del pH a la cosecha del cultivo en función de $\mathrm{pH}$ inicial testeado previo a la aplicación de los tratamientos de enmiendas. El pH fue cuantificado con la metodología suelo-agua 1:25. Los datos fueron sometidos a análisis de varianza al 5\% de probabilidades de error, y las medias fueron comparadas mediante prueba de Tukey al 5\%.

\section{Manejo específico del experimento}

Previo a la siembra las semillas fueron desinfectadas contra hongos e insectos del suelo, con Vitavax 300 PM (Carboxin 20\% + Captan 20\%) y Orthene 75 SP (Orthene 75\%), en dosis de 3 y $4 \mathrm{~g}$, respectivamente, disueltos en 0.5 litros de agua por cada kg de semilla. El control de arvenses se realizó en pre-emergencia con Linuron $50 \%+$ Pendimetalin $40 \%$ en dosis de $0,75 \mathrm{~kg}$ y 2,0 L ha-1 de cada producto, respectivamente. En pos-emergencia las arvenses fueron controladas con Fomesafen $25 \%+$ Haloxifop $40 \%$ en dosis de $0,8 \mathrm{~L} \mathrm{ha}^{-1}$ de cada producto. Se registró presencia de insectos defoliadores en una ocasión, los mismos que fueron controlados con aplicación de Diazinon $60 \%$ en dosis $0,75 \mathrm{~L} \mathrm{ha}^{-1}$. La presencia de insectos chupadores como moscas blancas (Bemicia tabaci), chicharritas (Empoasca kraemeri) y pulgones (Aphis sp.) fueron manejados con aplicaciones foliares de Imidacloprid $70 \%$ en dosis de $0,4 \mathrm{~L} \mathrm{ha}^{-1}$. Previo a la floración se presentó roya y otras machas foliares, que fueron manejadas con aperciones de Oxicarboxin $75 \%$ y Crorothalonil $75 \%$ en dosis $1 \mathrm{~g} \mathrm{~L}^{-1}$ y $5 \mathrm{~g} \mathrm{~L}^{-1}$ de cada producto, respectivamente. 


\section{RESULTADOS Y DISCUSIÓN}

La longitud radical, peso seco de planta y área foliar fueron significativamente influenciadas $(p<0,05)$ por las enmiendas aplicadas al suelo, donde la dolomita combinada con compost, gallinaza y humus alcanzaron los mayores promedios, en relación al tratamiento control, y las enmiendas con harina de rocas (Tabla 3). La longitud radical se incrementó en 11,12 y $16 \%$ con dolomita combinada con gallinaza, compost y humus, respectivamente, en relación a tratamiento control. El peso seco fue incrementado en 22, 27 y $31 \%$ con dolomita + gallinaza, compost y compost, respectivamente, en comparación al control. El área foliar se incrementó en un 42, 44 y $50 \%$ con los mismos tratamientos descritos, en contraste al tratamiento testigo. El tratamiento de fertilización convencional mostró un incremento del 9, 10 y $41 \%$ de longitud radical, peso seco de planta y área foliar, respectivamente, con relación al tratamiento control, pero menor a lo alcanzado por las combinaciones de dolomita, lo cual indica que el uso de enmiendas encalantes son determinantes para promover mayor desarrollo radical y crecimiento general del cultivo bajo condiciones de suelos ácidos.

Los resultados coinciden a los obtenidos por Shanka et al. (2017; 2018) que reportaron mayor acumulación de biomasa seca en plantas de frejol común tratadas con cal agrícola y compost en suelos ácidos Etiopía. Resultados similares fueron hallados por Effa et al. (2019) quienes reportaron mayor desarrollo radical, peso de biomasa y área foliar en plantas Pahseolus vulgaris y Vigna aconitifolia con aplicación de cal y abono orgánico en suelos ácidos de Nigeria.

Tabla 3. Efecto de varios tratamientos de enmiendas sobre la longitud radical, peso seco de planta y área foliar del frejol común establecido en suelo andisol ácido. Santo Domingo, Ecuador.

\begin{tabular}{llll}
\hline Enmiendas & Longitud radical $(\mathrm{cm})$ & Peso seco $(\mathrm{g})$ & Área foliar $\left(\mathrm{cm}^{2}\right)$ \\
\hline Humus + Harina de rocas & $19,67 \pm 0,54 \mathrm{bc}^{1 /}$ & $25,04 \pm 0,04 \mathrm{ab}$ & $1186,31 \pm 0.96 \mathrm{ab}$ \\
Humus + Dolomita & $22,11 \pm 0,16 \mathrm{a}$ & $31,90 \pm 0,21 \mathrm{a}$ & $1919,10 \pm 0,84 \mathrm{a}$ \\
Compost + Harina de rocas & $19,34 \pm 0,89 \mathrm{bc}$ & $24,56 \pm 0,50 \mathrm{ab}$ & $1353,52 \pm 0,80 \mathrm{ab}$ \\
Compost + Dolomita & $21,15 \pm 0,02 \mathrm{ab}$ & $30,45 \pm 0,08 \mathrm{ab}$ & $1733,32 \pm 0,98 \mathrm{ab}$ \\
Gallinaza + Harina de rocas & $20,22 \pm 0,24 \mathrm{abc}$ & $25,82 \pm 0,46 \mathrm{ab}$ & $1186,72 \pm 0,75 \mathrm{ab}$ \\
Gallinaza + Dolomita & $20,90 \pm 0,67 \mathrm{ab}$ & $28,25 \pm 0,12 \mathrm{ab}$ & $1665,68 \pm 0,56 \mathrm{ab}$ \\
Fertilizante compuesto & $20,33 \pm 0,22 \mathrm{abc}$ & $24,69 \pm 0,23 \mathrm{ab}$ & $1636,74 \pm 0,70 \mathrm{ab}$ \\
Control & $18,57 \pm 0,72 \mathrm{c}$ & $22,11 \pm 0,18 \mathrm{~b}$ & $964,48 \pm 0,74 \mathrm{~b}$ \\
\hline C.V. \% & 3,34 & 11,79 & 19,32 \\
p-valor ANAVA & $0,0005^{* *}$ & $0,0260^{*}$ & $0,0114 *$ \\
\hline
\end{tabular}

1/ Medias dentro de columnas con letras distintas, difieren estadísticamente según la prueba de Tukey al 5\% de probabilidades de error estándar. ${ }^{* *}$ Significativo al $1 \%$ de probabilidades de error; ${ }^{*}$ Significativo al $5 \%$ de probabilidades de error. 
Los componentes de rendimiento número de vainas, peso de 100 vainas y peso de 100 granos fueron significativamente afectados $(p<0,05)$ por los tratamientos de enmiendas evaluados, donde la dolomita mezclada con gallinaza, compost y humus alcanzaron los mayores valores, en relación al tratamiento control (Tabla 4). Las combinaciones de enmiendas orgánicas con harina de rocas y el tratamiento con fertilización convencional también superaron estadísticamente al tratamiento control, pero no a los tratamientos a base de dolomita (Tabla 4). La dolomita mezclada con gallinaza, compost y humus mostraron mayor efectividad en el incremento de vainas por planta con 24, 27 y $32 \%$, peso de 100 vainas con 9,11 y $12 \%$ y peso de 100 granos con 24, 27 y $29 \%$, en relación al tratamiento control, respectivamente para las tres variables.

Tabla 4. Efecto de enmiendas sobre el número de vainas planta-1, peso de 100 vainas y peso de 100 granos del frejol común establecido en suelo andisol ácido. Santo Domingo, Ecuador.

\begin{tabular}{lccc}
\hline Enmiendas & $N^{\circ}$ de vainas planta ${ }^{-1}$ & Peso de 100 vainas (g) & Peso de 100 granos (g) \\
\hline Humus + Harina de rocas & $10,78 \pm 0,63 \mathrm{~cd}$ & $243,41 \pm 0.45 \mathrm{ab}$ & $63,28 \pm 0,08 \mathrm{ab}$ \\
Humus + Dolomita & $14,42 \pm 0,52 \mathrm{a}$ & $259,33 \pm 0.92 \mathrm{a}$ & $70,00 \pm 0,15 \mathrm{a}$ \\
Compost + Harina de rocas & $11,22 \pm 0,41 \mathrm{bcd}$ & $235,00 \pm 0,46 \mathrm{ab}$ & $61,67 \pm 0,21 \mathrm{ab}$ \\
Compost + Dolomita & $13,33 \pm 0,88 \mathrm{ab}$ & $256,67 \pm 0,72 \mathrm{ab}$ & $68,12 \pm 0,40 \mathrm{a}$ \\
Gallinaza + Harina de rocas & $12,45 \pm 0,75 \mathrm{abc}$ & $243,14 \pm 0,48 \mathrm{ab}$ & $58,28 \pm 0,34 \mathrm{bc}$ \\
Gallinaza + Dolomita & $12,80 \pm 0,86 \mathrm{abc}$ & $250,36 \pm 0,63 \mathrm{ab}$ & $65,68 \pm 0,48 \mathrm{ab}$ \\
Fertilizante compuesto & $12,44 \pm 0,72 \mathrm{abc}$ & $253,33 \pm 0,56 \mathrm{ab}$ & $65,00 \pm 32 \mathrm{ab}$ \\
Control & $9,78 \pm 0,56 \mathrm{~d}$ & $228,30 \pm 0,32 \mathrm{~b}$ & $50,13 \pm 0,62 \mathrm{c}$ \\
\hline C.V. \% & 6,12 & 4,14 & 4,68 \\
p-valor ANAVA & $0,0001^{* *}$ & $0,0270 *$ & $0,0001^{* *}$ \\
\hline
\end{tabular}

1/ Medias dentro de columnas con letras distintas, difieren estadísticamente según la prueba de Tukey al 5\% de probabilidades de error estándar. ${ }^{* *}$ Significativo al $1 \%$ de probabilidades de error; ${ }^{*}$ Significativo al $5 \%$ de probabilidades de error.

El rendimiento de grano también fue influenciado significativamente $(p<0,05)$ por las combinaciones de enmiendas probadas, donde la dolomita combinada con gallinaza, compost y humus lograron los mayores rendimientos con 3100, 3407 y $3473 \mathrm{~kg} \mathrm{ha}^{-1}$, respectivamente, en contraste al tratamiento control que alcanzó $2243 \mathrm{~kg} \mathrm{ha}^{-1}$ (Figura 1). Lo anterior significó un incremento de rendimiento del 21,
28 y $30 \%$ con dolomita mezclada con gallinaza, compostyhumus, respectivamente, en relaciónal tratamiento testigo; mientras que con respecto al tratamiento de fertilización convencional que solo alcanzó $2958 \mathrm{~kg} \mathrm{ha}^{-1}$ de granos, los tratamientos a base de dolomita mezclados con gallinaza, compost y humus incrementaron el rendimiento de grano en un 5,13 y $15 \%$, respectivamente (Figura 1 ). 


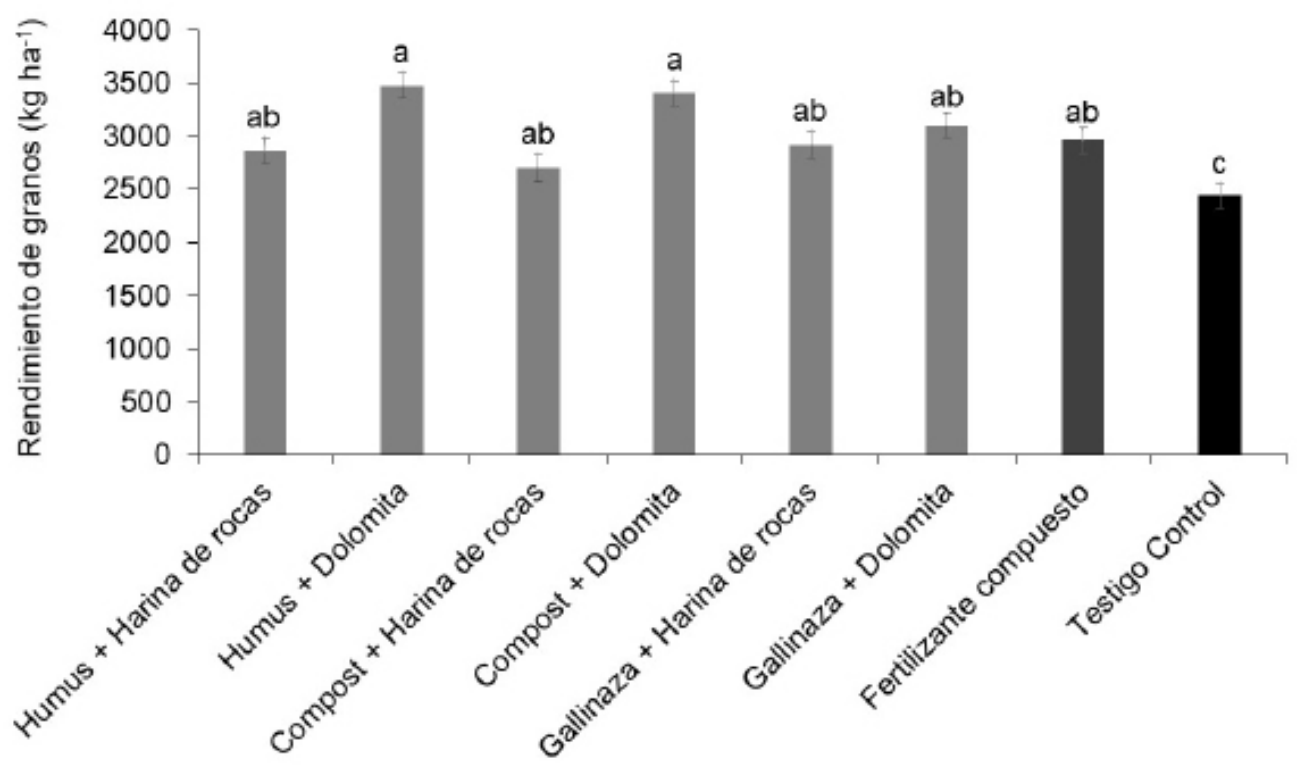

Figura 1. Efecto de las enmiendas sobre el rendimiento de granos del frejol común en un suelo andisol ácido. Santo Domingo, Ecuador. Cada barra representa la media de tres repeticiones. Barras con letras distintas difieren estadísticamente de acuerdo a la prueba de Tukey al 5\% de probabilidades de error.

Los resultados hallados de componentes de rendimiento y rendimiento de grano son cercanos a los obtenidos por Shanka et al. (2017) y Effa et al. (2019) quienes reportaron mayor producción de vainas por planta y de granos por hectárea en Phaseolus vulgaris y Vigna aconitifolia con aplicación de cal y abonos orgánicos en suelos ácidos de Etiopía y Nigeria, respectivamente. Por su parte, Sultana et al. (2019) incrementaron el rendimiento de grano en Vigna radiata en más del $100 \%$, en relación al tratamiento control, con aplicaciones combinadas de cal con estiércol de pollo y de corral, en suelo ácidos de las Ilanuras piemontanas del Himalaya.

En investigaciones realizadas por Verde et al. (2013) en soya establecida en suelos ácidos de Kenia, reportaron mayor rendimiento en tratamientos con enmiendas de cal mezcladas con estiércol de corral, donde el rendimiento de granos se incrementó hasta en $114 \%$ en relación al tratamiento control. Resultados similares fueron informados por Fekadu et al.
(2018) en el cultivo de haba, donde reportaron mayor rendimiento de granos en suelos ácidos de las tierras altas de Etiopía, con el uso de enmiendas cálcicas y abonos orgánicos como compost y estiércol de corral.

Los resultados evidencian que la cal dolomítica es determinante para mejorar la producción del cultivo de frejol común bajo condiciones de andisoles ácidos, independientemente de la enmienda orgánica con que se combine. Por otra parte, los resultados demuestran que si bien es cierto la fertilización convencional produjo mayores respuestas de crecimiento y rendimiento, con relación al tratamiento control, esta no superó a los tratamientos a base de dolomita (Tablas 3 y 4; Figura 1), lo cual sugiere que bajo condiciones de suelos andisoles, la práctica de encalado previamente a la fertilización debería ser efectuada.

En este sentido, Fekadu et al. (2018) reportaron rendimientos mayores de $1400 \mathrm{~kg} \mathrm{ha}^{-1}$ de granos en haba establecida en suelos ácidos, 
que recibió fertilización fosfatada combinada con compost y cal, en contraste a los $843 \mathrm{~kg}$ ha $^{-1}$ de granos que alcanzó el tratamiento solo con fertilización fosfatada. En experimentos realizados por Shanka et al. (2017; 2018) reportaron mayor efecto de la fertilización fosfatada sobre la nodulación y rendimiento del frejol cuando esta se realizó en combinación con cal y compost.

Todas las combinaciones de enmiendas utilizadas influenciaron significativamente $(p<0,05)$ el $\mathrm{pH}$ final del suelo, donde las enmiendas a base de dolomita mezcladas con gallinaza, compost y humus incrementaron el pH hasta 5,74; 5,76 y 5,85, respectivamente, con respecto al $\mathrm{pH}$ inicial que fue 4,90 (Figura 2). De forma similar, los tratamientos a base de harina de rocas combinados con gallinaza, compost y humus incrementaron el $\mathrm{pH}$ hasta 5,$28 ; 5,30$ y 5,35 , en su orden respectivo, con relación al $\mathrm{pH}$ inicial (Figura 2).
Las enmiendas dolomita mezcladas con gallinaza, compost y humus mostraron un efecto marcado en el incremento del $\mathrm{pH}$ del suelo, puesto que las tres combinaciones alcanzaron y superaron el valor de $\mathrm{pH}$ 5,5 (Figura 2), que ha sido considerado suficiente para precipitar el $\mathrm{Al}^{+3}$ en suelos tropicales ácidos, pues valores inferiores a 5,5 de $\mathrm{pH}$ contribuyen a la solubilización y liberación del $\mathrm{Al}^{+3}$ (Castro y Munevar, 2013; Rahman et al., 2018). La harina de roca en combinación con gallinaza, compost y humus, no mostraron el mismo efecto que las combinaciones con dolomita para alcanzar valores de $\mathrm{pH}$ necesarios para precipitar $\mathrm{Al}^{+3}$, sin embargo, es importante destacar que mejoraron el $\mathrm{pH}$ del suelo con respecto al testigo (Figura 2), y proporcionan importantes cantidades de minerales necesarios para mitigar los efectos tóxicos del $\mathrm{Al}^{+3}$ y estabilizar micronutrientes en suelos ácidos (Kim et al., 2016; Rahman et al., 2018).

\section{$\mathrm{pH}$ inicial (-) \\ $\mathrm{pH}$ necesario para precipitar $\mathrm{Al}^{+3}(---)$}

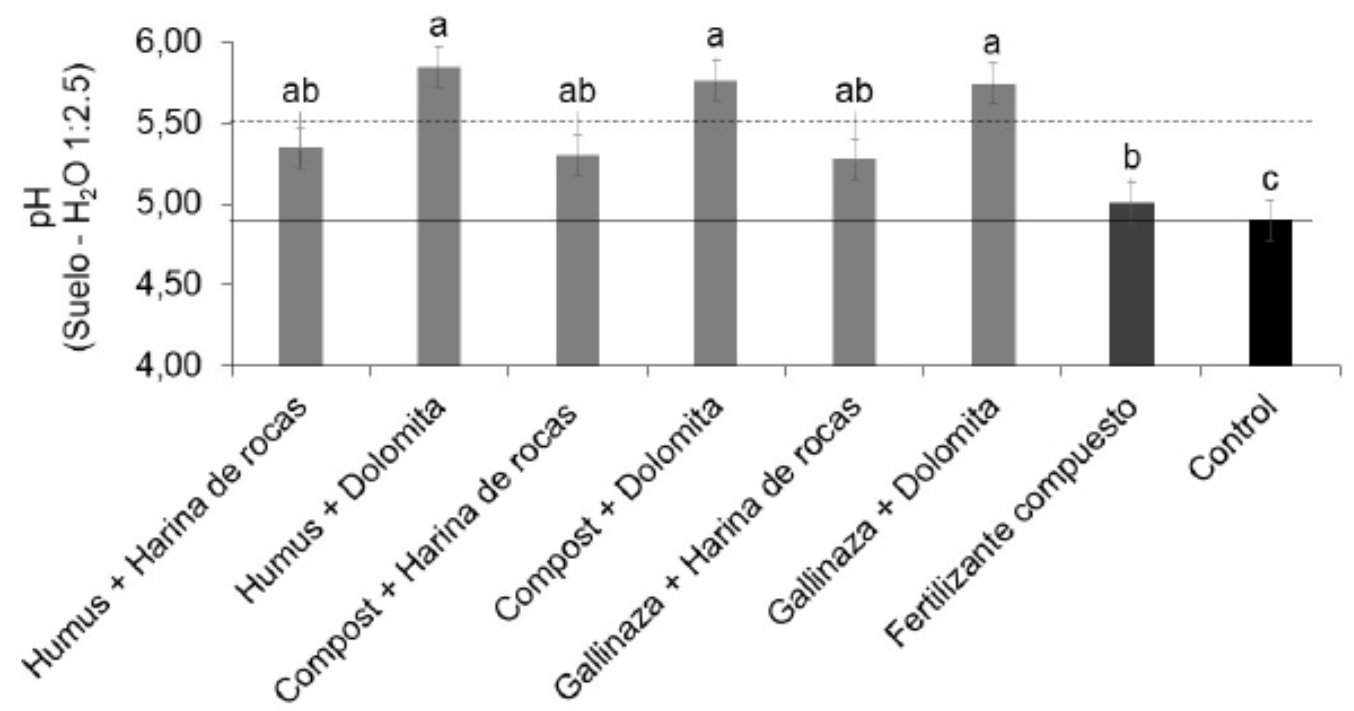

Figura 2. Efecto de las enmiendas sobre el $\mathrm{pH}$ final de un suelo andisol ácido. Santo Domingo, Ecuador, 2019. Cada barra representa la media de tres repeticiones ( \pm error estándar). Barras con letras distintas difieren estadísticamente de acuerdo a la prueba de Tukey al 5\% de probabilidades de error. 
Los resultados encontrados en cuanto a cambios de $\mathrm{pH}$, guardan similitud a los descritos por Verde et al. (2013) quienes lograron elevar el $\mathrm{pH}$ de suelos ácidos de Kenia, de 5,05 hasta 6,64 y 5,79 con aplicaciones combinadas de cal, estiércol y súper fosfato triple. En condiciones similares de suelos ácidos de Kenia, Otieno et al. (2018) lograron incrementar el $\mathrm{pH}$ hasta 7,36 y 6,06 con respecto a los $\mathrm{pH}$ iniciales de 4,8 y 4,1 , respectivamente, con aplicaciones de estiércol y cal, en dos localidades de este país. Resultados cercanos fueron alcanzados por Bekele et al. (2018), quienes reportaron valores de $\mathrm{pH}$ superiores a 5,5 en relación al $\mathrm{pH}$ inicial de 4,80, lo cual fue logrado con aplicaciones simples y combinadas de cal y enmiendas orgánicas. Por su parte, Castro y Guerrero (2018) demostraron la efectividad de varios materiales encalantes en el incremento del $\mathrm{pH}$ de un suelo ácido de Colombia, donde las escorias básicas, dolomitas y mezclas con gallinaza superaron el valor de 5,5 de $\mathrm{pH}$, en relación al $\mathrm{pH}$ inicial de 4,86. Finalmente, Yao et al. (2019) lograron incrementar el $\mathrm{pH}$ de un suelo ácido desde 5,49 hasta 6,55 con aplicaciones combinadas de cal y biochar.

El efecto de la dolomita mezclada con abonos orgánicos fue eficiente para reducir la acidez del suelo, debido a que es ampliamente aceptado que cuando se agrega cal a los suelos ácidos que contienen altas concentraciones de $\mathrm{Al}^{3+} \mathrm{y} \mathrm{H}^{+}$, se disocia en iones $\mathrm{Ca}^{2+}$ y $\mathrm{OH}^{-}$, donde este último reacciona con hidrógeno e iones $\mathrm{Al}^{3+}$, formando hidróxido $\mathrm{Al}^{3+}$ y agua; de ese modo aumenta el $\mathrm{pH}$ en la solución del suelo (Ameyu et al. 2019). Sin embargo, es de destacar que la efectividad del encalado también depende del tipo de cal aplicada, dosis, método de aplicación y especie cultivada. En este sentido, se ha estimado de forma general que la aplicación de $\mathrm{CaO}$, $\mathrm{CaCO} 3, \mathrm{Ca}(\mathrm{OH}) 2$ y $\mathrm{CaMg}(\mathrm{CO} 3) 2$ aumentó el rendimiento en 13,$2 ; 34,3 ; 29,2$ y $66,5 \%$, respectivamente (Li et al., 2018).

El efecto de estiércoles y materias orgánicas como materiales encalantes para incrementar $\mathrm{pH}$ en suelos ácidos, ha sido atribuido a la amortiguación de bicarbonatos y ácidos orgánicos presentes en el estiércol, además del intercambio de protones entre el suelo y los sistemas de amortiguamiento de los materiales orgánicos (Whalen et al., 2000; Wong y Swift, 2001). Por otra parte, ha sido propuesto que la adición de materiales orgánicos como compost y biochar puede ayudar a reducir la volatilización del amoniaco, retención del nitrato y por tanto la elevación del pH (Kammann et al., 2015; Hagemann et al., 2017). Además, también es posible que el material de compost pueda contener compuestos de carbonato debido a la reacción entre el dióxido de carbono (CO2) y los metales minerales, lo que lleva a un aumento del pH (Castán et al., 2016).

El efecto alcalinizante de enmiendas orgánicas a menudo se ha registrado como uno de los principales mecanismos responsables del aumento de los rendimientos de los cultivos en suelos ácidos, además del mejoramiento de otras propiedades físicas, químicas y biológicas del suelo, que favorecen el desarrollo radical (Kätterer et al., 2019; Wang et al., 2019). En este mismo contexto, se ha comprobado que la aplicación de enmiendas cálcicas además de reducir la acidez del suelo, genera otros beneficios directos como el incremento en la concentración de bases, disponibilidad de nutrientes, fijación simbiótica de $\mathrm{N}_{2}$ y reducción de iones tóxicos para las plantas como $\mathrm{Al}$, Fe y $\mathrm{Mn}$, lo que finalmente se traduce en el incremento del crecimiento radical y rendimiento de los cultivos ( $\mathrm{Li}$ et al., 2018; Ameyu et al. 2019). 


\section{CONCLUSIONES}

Las enmiendas aplicadas fueron efectivas para incrementar el crecimiento y rendimiento del cultivo de frejol común bajo condiciones de andisoles ácidos. Las enmiendas basadas en dolomita y mezcladas con gallinaza, compost y humus fueron más eficaces para reducir la acidez del suelo e incrementar el $\mathrm{pH}$ a niveles considerados suficientes para precipitar al $\mathrm{Al}^{3+}$. La harina de rocas mezclada con gallinaza, compost y humus no alcanzo niveles suficientes de $\mathrm{pH}$ para precipitar el $\mathrm{Al}^{3+}$, pero mostraron efectividad para incrementar el crecimiento y rendimiento del cultivo de frejol común. Se sugiere que la aplicación de enmiendas encalantes deber ser una práctica implementada en suelos andisoles ácidos con la finalidad de potenciar el efecto de la fertilización en el cultivo de frejol común.

\section{Conflicto de Intereses}

Los autores declaran que es un trabajo orginal y no existío conflicto de intereses de ningún tipo en la elaboración y publicación del manuscrito.

\section{REFERENCIAS}

Alemu, H. 2017. Review Paper On Breeding Common Bean (Phaseolus Vulgaris L.) Genotypes For Acidic Soil Tolerance. International Journal of Advanced Research and Publications 1(3): $39-46$.

Ameyu, T. 2019. A Review on the Potential Effect of Lime on Soil Properties and Crop Productivity Improvements. Journal of Environment and Earth Science 9(2): $17-23$.

Bekele, A., Kibret, K., Bedadi, B., Yli-Halla, M. and Balemi, T. 2018. Effects of Lime, Vermicompost, and Chemical P Fertilizer on Selected Properties of Acid Soils of Ebantu District, Western Highlands of Ethiopia. Applied and Environmental Soil Science 8178305: 1 - 13.
Castán, E., Satti, P., González-Polo, M., Iglesias, M.C. and Mazzarino, M.J., 2016. Managing the value of composts as organic amendments and fertilizers in sandy soils. Agric. Ecosyst. Environ. 224: 29-38.

Castro, H. y Munevar, O. 2013. Mejoramiento químico de suelos ácidos mediante el uso combinado de materiales encalantes.

Revista U.D.C.A Actualidad \& Divulgación Científica 16 (2): 409 - 416.

Castro, H. y Guerrero, J. 2018. Evaluación de materiales de encalado mediante pruebas de incubación en un oxisol de la altillanura colombiana. Revista de Ciencias Agrícolas 34(2):14-26.

Dida, G. and Etisa, D. 2019. Effect of Lime and Compost Application on the growth and yield of Common Bean (Phaseolus Vulgaris L.): A Review. Advances in Oceanography \& Marine Biology 1(3): 1 -9 .

Effa, E., Mofunanya, A. and Ngele, B. 2019. Effect of Organic Manure and Agricultural Lime on Growth and Yield of Two Bean Varieties. International Journal of Plant \& Soil Science 30(4): 1-18.

Fekadu, E., Kibret, K., Melese, A. and Bedadi, B. 2018. Yield of faba bean (Vicia faba L.) as affected by lime, mineral $\mathrm{P}$, farmyard manure, compost and rhizobium in acid soil of Lay Gayint District, northwestern highlands of Ethiopia. Agric \& Food Secur 7(16): $1-11$.

Habtamu, A. 2017. Review Paper On Breeding Common Bean (Phaseolus Vulgaris L.) Genotypes For Acidic Soil Tolerance. International Journal of Advanced Research and Publications 1(3): $39-46$. 
Hagemann, N., Joseph, S., Schmidt, H., Kammann, C., Harter, J., Borch, T., Young, R., Varga, K., Taherymoosavi, S., Elliott, K., McKenna, A., Albu, M., Mayrhofer, C., Obst, M.; Conte, P., Dieguez, A., Orsetti, S., Subdiaga, E., Behrens, S. and Kappler, A. 2017. Organic coating on biochar explains its nutrient retention and stimulation of soil fertility. Nature Communications 8(1089): 1 - 11 .

Kammann, C., Schmidt, H., Messerschmidt, N., Linsel, S., Steffens, D., Müller, C., Koyro, H., Conte, P. and Joseph, S. 2015. Plant growth improvement mediated by nitrate capture in co-composted biochar. Scientific Reports 5(11080): 1 - 12.

Kätterer, T., Roobroeck, D., Andrén, O., Kimutai, G., Karltun, E., Kirchmann, H., Nyberg, G., Vanlauwe, B. and de Nowina, K.R., 2019. Biochar addition persistently increased soil fertility and yields in maize-soybean rotations over 10 years in sub-humid regions of Kenya. Field Crops Research 235: 18-26.

Kim, M., Min, H., Lee, S. and Kim, J. 2016. The Effects of Various Amendments on Trace Element Stabilization in Acidic, Neutral, and Alkali Soil with Similar Pollution Index. PLoS ONE 11(11): e0166335. doi:10.1371/journal.pone.0166335.

Lal, N. 2016. Effects of Acid Rain on Plant Growth and Development. e-Journal of Science and Technology 11(5): 85 - 108.

Legesse, H., Nigussie, R., Gebeyehu, S., Bultosa, G. and Mekbib, F. 2013. Response to Soil Acidity of Common Bean Genotypes (Phaseolus vulgaris L.) Under Field Conditions at Nedjo, Western Ethiopia. Science, Technology and Arts Research Journal 2(3): 03-15.
Li, Y., Cui, S., Chang, S. and Zhang, Q. 2018. Liming effects on soil $\mathrm{pH}$ and crop yield depend on lime material type, application method and rate, and crop species: a global meta-analysis. Journal of Soils and Sediments 19: 1393-1406.

Lunze L., Kimani P., Ngatoluwa, R., Rabary B., Rachier G., Ugen M., Ruganza V. and Awad Elkarim E. 2007. Bean improvement for low soil fertility in adaptation in Eastern and Central Africa. In: Bationo, A., Waswa, B., Kihara, J. \& Kimetu J. (Eds.) Advances in integrated soil fertility management in sub- Saharan Africa: Challenges and Opportunities, pp. 325 - 332. Springer, Dordrecht, The Netherlands.

MAG (Ministerio de Agricultura y Ganadería). 2017. Boletín Situacional de Fréjol Tierno y Seco. Sistema de Información Pública Agropecuaria (SIPA). Quito, EC. 8 p.

Mite, F., Medina, L. y Espinosa, J. 2009. Efecto de la corrección del $\mathrm{pH}$ en el rendimiento de piña en suelos volcánicos. Informaciones Agronómicas 73: 1 - 5 .

Otieno, H., Cheminingwa, G. and Zingore, S. 2018. Effect of Farmyard Manure, Lime and Inorganic Fertilizer Applications on Soil pH, Nutrients Uptake, Growth and Nodulation of Soybean in Acid Soils of Western Kenya. Journal of Agricultural Science 10(4): 199 - 208.

Peralta, E., Murillo, A., Mazón, N. and Pinzón, J., Villacrés, E. 2013. Manual Agrícola de fréjol y otras leguminosas. Cultivos, variedades y costos de producción. Publicación Miscelánea \# 135. Tercera Edición. Programa Nacional de Leguminosas y Granos Andinos. Estación Experimental Santa Catalina. INIAP, Quito, Ecuador. 70 p. 
Petry, N., Boy, E., Wirth, J. and Hurrell, R. 2015. Review: The Potential of the Common Bean (Phaseolus vulgaris) as a Vehicle for Iron Biofortification. Nutrients 7: 11441173.

Pérez, A., Martínez, E., Vélez, L. y Cotes, J. 2013. Acumulación y Distribución de Fitomasa en el Asocio de Maíz (Zea mays L.) y Fríjol (Phaseolus vulgaris L.). Rev. Fac.Nal.Agr. Medellín 66(1):6865-6880.

Rangel, A., Rao, I. and Horst, W. 2007. Spatial aluminium sensitivity of root apices of two common bean (Phaseolus vulgaris L.) genotypes with contrasting aluminum resistance. J. Exp. Bot. 58: 3895-3904.

Rahman, M., Lee, S., Ji, H., Kabir, A., Jones, C. and Lee, K. 2018. Importance of Mineral Nutrition for Mitigating Aluminum Toxicity in Plants on Acidic Soils: Current Status and Opportunities. International Journal of Molecular Sciences 19(3073).

Shanka, D., Dechassa, N., Gebeyehu, S. and Elias, E. 2017. Yield Response of Common Bean to Phosphorus, Lime and Compost Application at Areka, Southern Ethiopia. Journal of Science and Sustainable Development 5(1): 37-55.

Shanka, D., Dechassa, N., Gebeyehu, S. and Elias, E. 2018. Dry matter Yield and Nodulation of Common Bean as Influenced by Phosphorus, Lime and Compost Application at Southern Ethiopia. Open Agriculture 3: 500-509.

Sultana, B., Mian, M., Jahiruddin, M., Rahman, M., Siddique, N. and Sultana, J. 2019. Amendment of Acidic Soil with Lime and Manure for Enhancing Fertility, Nutrient Uptake and Yield of Wheat-MungbeanMonsoon Rice in the Old Himalayan Piedmont Plain. Asian Journal of Soil Science and Plant Nutrition 4(2): 1-26.
Verde, B., Danga, B. and Mugwe, J. 2013. Effects of manure, lime and mineral $\mathrm{P}$ fertilizer on soybean yields and soil fertility in a humic nitisol in the Central Highlands of Kenya. International Journal of Agricultural Science Research 2(9): 283-291.

Wang, Y., Villamil, M., Davidson, P. and Akdeniz, N. 2019. A quantitative understanding of the role of co-composted biochar in plant growth using meta-analysis. Science of the Total Environment 685: 741-752.

Wong, M. and Swift, R. 2001. Application of fresh and humified organic matter to ameliorate soil acidity. In: Understanding and Managing Organic Matter in Soils, Sediments, and Waters. R.S. Swift and K.M. Spark (Eds). 235 - 242 pp.

Whalen, J., Chang, C., Clayton, G. and Carefoot, J. 2000. Cattle Manure Amendments Can Increase the $\mathrm{pH}$ of Acid Soils. Soil Sci. Soc. Am. J. 64:962-966.

Yang, Q., Gan, R., Ge, Y. Zhang, D. and Corke, H. 2018. Polyphenols in Common Beans (Phaseolus vulgaris L.): Chemistry, Analysis, and Factors Affecting Composition. Comprehensive Reviews in Food Science and Food Safety 17: 1518 -1539 .

Yao, L., Yu, X., Zhang, X., Wang, D., Zhao, X., Li, Y., He, Z., Kang, L., Li, X., Liu, D., Xiao, Q. and Guo, Y. 2019. Responses of Phaseolus calcaltus to lime and biochar application in an acid soil. Peer J 7(e6346): 1 - 25. 